\title{
Newborn screening for Duchenne muscular dystrophy: a psychosocial study
}

\author{
E P Parsons, A J Clarke, K Hood, E Lycett, D M Bradley
}

Arch Dis Child Fetal Neonatal Ed 2002;86:F91-F95

\begin{abstract}
Objective: To evaluate the psychosocial implications of newborn screening for Duchenne muscular dystrophy.

Design: Prospective psychosocial assessment.

Setting: Primary care.

Respondents: Study: (a) families of an affected boy identified by screening $(n=20)$; $(b)$ families of a boy with a transient screening abnormality $(n=18)$. Control: (a) families of a boy with a later clinical diagnosis $(n=16)$; (b) random sample of mothers of boys aged 6-9 months $(n=43)$.

Interventions: Questionnaires and semistructured interviews.

Main outcome measures: Attitudes to newborn screening and impact of screening on mother-baby relationship, anxiety/wellbeing, and reproductive patterning within families of an affected boy.

Results: Most families of an affected boy were in favour of newborn screening on the grounds of reproductive choice and time to prepare emotionally and practically. There was no evidence of any long term disruption to the mother-baby relationship. Anxiety levels for the screened group were slightly above threshold but returned to normal during the period of the study. There was no evidence, from anxiety or wellbeing scores, that the transient group had suffered any disadvantage. Although the profile of the screened and later clinically diagnosed cohorts was similar after diagnosis, when boys from the screened cohort were 4 years old and more socially aware, their profile was more positive. There was evidence that reproductive patterning had been modified, and four fetuses carrying a mutation causing Duchenne muscular dystrophy were terminated.

Conclusion: A case can be made for newborn screening provided that the test is optional, a rigorous protocol for service delivery is used, and an infrastructure providing continuing support is in place.
\end{abstract}

See end of article for authors' affiliations

Correspondence to:

Dr Parsons, School of

Nursing and Midwifery

Studies, UWCM, Heath

Park, Cardiff CF14 4XN,

Wales, UK;

parsonsep@cardiff.ac.uk

Accepted

19 October 2001
$\mathrm{D}$ uchenne muscular dystrophy (DMD) is a lethal genetic disease which is traditionally diagnosed symptomatically, the mean age of detection being between 4 and 5 years. ${ }^{1-3}$ It has been recognised that this diagnostic process has two major disadvantages: firstly, it is often prolonged ${ }^{24}$ with no evidence of any improvement over the past 20 years, ${ }^{5}$ and secondly, further affected boys may be born before the diagnosis is made. ${ }^{2}$ Two alternative methods of earlier diagnosis have been proposed. The first is to screen boys at 18 months. ${ }^{6}$ This approach only provides reproductive choice after 18 months and is difficult to implement at the primary healthcare level. ${ }^{7}$ The second method is to screen boys in the newborn period. Newborn screening became feasible in 1975 with the development of an assay for creatine kinase in dried blood samples, ${ }^{8}$ but, although several centres undertake screening, ${ }^{9}$ it is still highly controversial. It has been argued that choice in future pregnancies does not outweigh the potential disadvantages of screening for an untreatable disease. In particular, health professionals have been concerned about any effect on the early mother-baby relationship, ${ }^{10}{ }^{11}$ although these concerns have not been substantiated by research based evidence.

We report a long term psychosocial follow up of families identified during a newborn screening programme for DMD. We examine the advantages and disadvantages experienced by families with an affected boy and those whose boy had a transient abnormality - that is, a raised creatine kinase activity on screening but a normal level in a venous blood sample at 6 weeks of age.

The research took place between July 1990 and March 1998. On the basis of the evidence provided to the health authorities in Wales, newborn screening has been funded since April 1998. Details of the laboratory process, the protocol developed for service delivery, and the attitude of health professionals and families who opted out of screening have been reported elsewhere. ${ }^{92-14}$

\section{METHODS}

In 1990 an "opt in" newborn screening programme for DMD was introduced in Wales to evaluate the feasibility of screening and to assess the psychosocial implications of such an early diagnosis. Parents were given information (verbally and in writing) about each of the diseases screened for in the newborn period and the management and treatment available for them. In the case of the DMD test, the information leaflet stated that it was an extra test that parents could choose to have and that there was no cure for the disease.

During the eight year research period, 139796 samples from boys were received in the laboratory, and 7531 (5.4\%) families declined the DMD test. The parents' decision was not recorded by the midwife in a further $3171(2.3 \%)$ cases. The remaining 129094 boys were tested (uptake rate of 92.3\%). There were 43 abnormal screening results; 22 abnormal tests (1:5867) were confirmed on a subsequent venous blood sample. Of these 22 confirmed cases, two families were not approached because they had a known family history of DMD, while the remaining 20 families were invited and agreed to join the psychosocial research. These boys have all been confirmed as having DMD, either by muscle biopsy or a combination of mutation analysis and clinical symptoms. There were 15 families whose sons had reached 4 years of age by the end of the research, all of whom took part in the second interview.

To assess the implications of newborn screening, we collected data from four populations. 


\section{Study populations}

Families of an affected boy identified by screening

(screened cohort $\mathrm{n}=20$ )

Case studies were constructed for each family from the time of the screening test to the boy's 4th birthday. There were four main data sources: (a) questionnaire and semistructured interview with the family at 9 months of age (first interview); (b) informal home visits by the researcher at 2 and 3 years of age; (c) questionnaire and semistructured interview with the family when the boy was 4 years old (second interview); $(d)$ a questionnaire completed by the family's health visitor during the first six months. Further details of the research methods have been published elsewhere. ${ }^{15}$

\section{Families of a boy with a transient screening abnormality (transient cohort $\mathrm{n}=18$ )}

Data were collected from one semistructured interview and questionnaire two to three months after the normal result had been given. There were 21 transient abnormalities (1:6147), with three families not being contacted because resampling had already been performed as part of continuing medical interventions. The remaining 18 families agreed to join the study.

Families of a boy who experienced a later clinical diagnosis (later clinically diagnosed cohort $n=16$ )

A group of families from Wales and the South West of England were interviewed and completed a questionnaire in the year after diagnosis. This group was used as a control for the screened cohort.

The mean (SE) age of diagnosis for this cohort was 50 (6.4) months (range 18-101), with mothers reporting a mean (SE) delay of 23 (4.3) months (range 1-48) between raising their concerns with a health professional and diagnosis.

\section{A control group from the general population (general population cohort $n=43$ )}

A random sample of mothers with baby boys aged 6-9 months $(\mathrm{n}=108)$ drawn from newborn screening records were invited to complete a postal questionnaire. A total of 43 questionnaires were returned ( $40 \%$ response rate). This cohort provided control data for the screened and transient cohort with boys aged 6-9 months.

\section{Measures}

Attitudes to screening

A structured question was asked with the options: in favour, against, or undecided. These data were collected from all cohorts except the general population. In addition, during the interview, mothers from the transient cohort were asked whether they had any lingering doubts about the normal test result and whether they would permit screening of another baby for DMD.

\section{Mother-baby relationship}

Three measures were used to assess the relationship.

(1) FIRST score: assessments made by health visitor of mother's responses to baby on five dimensions: feeding, interest, response, speech, and touch. ${ }^{16}$ Responses were assessed before the query about DMD was raised, immediately after, and one month later. Each dimension was scored from 0 to 2 giving a cumulative score ranging from 0 to 10 . These data were collected from the screened and transient cohorts.

(2) Rejection/protection index: mothers were asked to score five statements, one of which explored potential rejection ("I feel I want to run away and leave him"), and the others explored potential overprotection-for example, feeling protective, wanting to hug, watching closely, and not trusting him
Table 1 Attitudes to screening of the screened, transient, and later traditionally diagnosed cohorts

\begin{tabular}{lllll}
\hline & $\begin{array}{l}\text { Screened } \\
\text { 1st } \\
\text { interview } \\
(n=20)\end{array}$ & $\begin{array}{l}\text { Transient } \\
(n=18)\end{array}$ & $\begin{array}{l}\text { Screened } \\
\text { 2nd } \\
\text { interview } \\
(n=15)\end{array}$ & $\begin{array}{l}\text { Later } \\
\text { clinically } \\
\text { diagnosed } \\
(n=16)\end{array}$ \\
\hline In favour & $17(85 \%)$ & $16(89 \%)$ & $13(87 \%)$ & $14(88 \%)$ \\
Against & $1(5 \%)$ & $1(5.5 \%)$ & $2(13 \%)$ & $1(6 \%)$ \\
Undecided & $2(10 \%)$ & $1(5.5 \%)$ & $2(6 \%)$ \\
Total & $20(100 \%)$ & $18(100 \%)$ & $15(100 \%)$ & $16(100 \%)$ \\
\hline & & & & \\
\hline
\end{tabular}

with others. Each statement was scored 1 (strongly disagree) to 5 (strongly agree). These data were collected from all cohorts.

(3) Baby/child adjectives: Green et al ${ }^{17}$ have argued that an adjective checklist is a useful tool to evoke mothers' descriptions of their babies. Mothers were given a list of 20 adjectives and asked to select as many as they thought described their son. The adjectives were: doing well, healthy, strong, frail, little, contented, placid, good, responsive, alert, demanding, difficult, miserable, fretful, great, lovely, fun, cuddly, handful, exhausting. These data were collected from all cohorts.

\section{Anxiety and wellbeing}

Mothers completed the six item short form of the State scale, ${ }^{18}{ }^{19}$ which has a range of 6-24 with a normal range of 9-12. These data were collected from all cohorts except the general population. The General Health Questionnaire (GHQ) $12^{20}$ has a range of $0-12$ with a normal threshold of $2-3$. These data were collected from all cohorts.

\section{Reproductive patterning}

Details of the subsequent reported reproductive patterning of the screened cohort and their extended family were collected.

\section{Statistical analysis}

Data were analysed using SSPS for Windows version 10. Statistical analysis was carried out using Kruskal-Wallis analysis of variance, $t$ test, Mann-Whitney, Wilcoxon, and Fisher's tests as appropriate. Indications of the impact of multiple comparisons are given where appropriate using the Bonferroni correction.

\section{RESULTS}

\section{Attitudes to screening}

Table 1 shows attitudes to screening for the screened, transient, and later clinically diagnosed cohorts.

Screened cohort $(n=20)$

Families gave two main reasons for favouring newborn screening: reproductive choice and time to come to terms with the diagnosis before their son was aware, to plan housing and schooling, begin early physiotherapy, and make good memories. During their interviews, the two families who had marked their questionnaires "undecided" described how ambivalent they were, one minute in favour of screening, the next against. The family who were opposed to screening felt that they had not been given sufficient information and regretted the earlier diagnosis.

Transient cohort ( $\mathrm{n}=18$ )

The one mother against screening said she had regretted having the test and would have refused it had she realised that the disease was untreatable. This mother, and two others, said they would not allow testing of another baby. Two other mothers expressed some doubts about the normal result; for 
Table 2 FIRST scores for screened and transient cohorts

\begin{tabular}{llll}
\hline & Before query & After query & $\begin{array}{l}\text { One month } \\
\text { later }\end{array}$ \\
\hline Screened $(n=18)$ & $10(8-10)$ & $10(8-10)$ & $10(9-10)$ \\
Transient $(n=13)$ & $9(7-10)$ & $9(7-10)$ & $10(9-10)$ \\
\hline
\end{tabular}

Values are median (range).

FIRST, Feeding, interest, response, speech, and touch

one these concerns were understandable because a nonfamilial relative had an affected son. Both mothers were in favour of screening and would have another baby tested.

Later clinically diagnosed cohort $(n=16)$

Of the 16 families in this cohort, 14 said that they would have preferred newborn screening. There were four main reasons: the ability to plan for the future, earlier physiotherapy, to avoid diagnostic delay, and avoid misunderstanding their son's early symptoms. Several mothers felt that they would have coped better knowing when he was younger: one said "not knowing, you are just in a fool's paradise". One mother was against and one was undecided because, had she gone on to have a second affected boy, she would have been in favour.

\section{Mother-baby relationship}

\section{FIRST scores}

Table 2 shows the median scores and ranges for the screened and transient cohorts. There was only one case (screened cohort) where the health visitor noted a short term change in baby handling, with his mother not as free in handing the baby to visitors. One month after the query, all scores had either returned to the initial level or increased.

A significant increase was noted (Wilcoxon) for both cohorts in the scores one month after the query compared with those before the query (screened group, $\mathrm{z}=2.53$, $\mathrm{p}<0.05^{*}$; transient group, $\left.\mathrm{z}=2.71, \mathrm{p}<0.01^{*}\right)$ and immediately after the query (screened group, $\mathrm{z}=2.13, \mathrm{p}<0.05$; transient group, $\left.\mathrm{z}=2.53, \mathrm{p}<0.05^{*}\right)(*$ indicates that significance was retained after Bonferroni correction).

\section{Rejection/protection statements}

No significant difference was found between any of the cohorts on the statement about feelings of "wanting to run away" ( $t$ test, Wilcoxon, and Mann-Whitney). There were no mothers in the screened or transient cohort who agreed or strongly agreed with the statement, whereas there were three mothers in the general population cohort who did. For boys aged 6-9 months, a Kruskal-Wallis analysis of variance (nonparametric) found no differences between the three cohorts on any of the protection statements. Similarly the MannWhitney test found no differences between the screened group (second interview) and the later clinically diagnosed cohort when the boys were 4 years or older. There was, however, a significant difference found on the "watch" statement between first (3.3) and second (4.2) interview for the screened cohort $(\mathrm{z}=2.48, \mathrm{p}<0.05$; Wilcoxon $)$ and between the first (3.3) interview and later clinically diagnosed cohort (4.3) $(z=2.64, p<0.05$; Mann-Whitney), but none of these remained significant after Bonferroni's correction for multiple comparisons.

\section{Baby/child adjectives}

We analysed the frequency with which a particular adjective was chosen to describe their son by one cohort compared with another. We found no between cohort differences on any of the seven negative descriptors: frail, little, miserable, fretful, exhausting, difficult, or demanding or on four of the positive
Table 3 Baby adjectives used by mothers of boys 6-9 months old

\begin{tabular}{llll}
\hline Adjective & $\begin{array}{l}\text { General } \\
\text { population } \\
(\mathrm{n}=43)\end{array}$ & $\begin{array}{l}\text { Screened and } \\
\text { transient }(\mathrm{n}=27)\end{array}$ & $\begin{array}{l}\text { p Value } \\
\text { (Fisher's) }\end{array}$ \\
\hline Doing well & $38(88)$ & $17(63)$ & 0.02 \\
Healthy & $37(86)$ & $23(85)$ & 1.00 \\
Strong & $37(86)$ & $20(74)$ & $\mathrm{NS}$ \\
Responsive & $35(81)$ & $23(85)$ & $\mathrm{NS}$ \\
Alert & $41(95)$ & $20(74)$ & 0.02 \\
Great & $37(86)$ & $12(44)$ & $<0.001^{*}$ \\
Fun & $41(95)$ & $14(52)$ & $<0.001^{*}$ \\
Cuddly & $27(63)$ & $25(93)$ & $0.005^{*}$ \\
Handful & $20(47)$ & $3(11)$ & $0.003^{*}$ \\
\hline
\end{tabular}

Values in parentheses are percentages. Note: the screened cohort $1 \mathrm{st}$ interview and the transient cohort have been combined because of small numbers.

*Remain significant after Bonferroni's correction for multiple comparisons.

Table 4 Child adjectives used by mothers of boys 4 years or older

\begin{tabular}{llll}
\hline Adjective & $\begin{array}{l}\text { Screened (2nd } \\
\text { interview) } \\
(\mathbf{n}=15)\end{array}$ & $\begin{array}{l}\text { Later clinically } \\
\text { diagnosed } \\
(\mathbf{n}=16)\end{array}$ & $\begin{array}{l}\text { p Value* } \\
\text { (Fisher's) }\end{array}$ \\
\hline Doing well & $14(93)$ & $13(81)$ & NS \\
Healthy & $8(53)$ & $7(44)$ & NS \\
Strong & $8(53)$ & $5(31)$ & NS \\
Responsive & $10(67)$ & $4(25)$ & 0.03 \\
Alert & $11(73)$ & $7(44)$ & NS \\
Great & $11(73)$ & $5(31)$ & 0.03 \\
Fun & $15(100)$ & $12(75)$ & NS \\
Handful & $6(40)$ & $3(19)$ & NS
\end{tabular}

*None of these remain significant after Bonferroni's correction for multiple comparisons.

descriptors: contented, placid, good, or lovely. Tables 3 and 4 show usage of the remaining nine descriptors. Mothers from the screened and transient cohort, with boys aged 6-9 months old, were less likely than mothers in the general population to choose doing well, alert, great, fun, and handful. They were, however, more likely to choose cuddly. There were three changes over time noted for the screened cohort (Wilcoxon), with mothers by second interview more likely to choose doing well $(\mathrm{z}=2.24, \mathrm{p}<0.05)$, fun $(\mathrm{z}=2.65, \mathrm{p}<0.01)$, and handful $(z=2.83, p<0.01)$. The later clinically diagnosed cohort scored lower on all nine positive adjectives than the screened cohort (second interview). Differences on responsive and great were individually significant but did not remain after Bonferroni's correction for multiple comparisons.

\section{Anxiety and wellbeing}

A one way analysis of variance comparison showed no significant between cohort differences on either the anxiety or the wellbeing measure, although levels of anxiety in the screened cohort at 6-9 months of age (13.7) and later clinically diagnosed cohort at 4 years or older (13.1) were slightly above the normal threshold. The GHQ scores for all mothers with boys $6-9$ months old were slightly above threshold, as were those for the later clinically diagnosed cohort.

\section{Reproductive patterning}

The index and extended families, from the screened cohort, notified the Institute of Medical Genetics about 27 subsequent pregnancies. There were four social terminations, the relation of which to the diagnosis is unclear, and 19 of the remaining $23(83 \%)$ were tested or identified as being at minimal risk. The outcome of these 19 pregnancies was: seven healthy boys, 
Table 5 Potential advantages of a newborn screening diagnosis

\begin{tabular}{ll}
\hline 1. Reproductive choice & \\
Counselling/prenatal testing & $19 / 23$ \\
Changed intentions & $16 / 20$ \\
2. Time & $17 / 20$ \\
To prepare emotionally when son is less aware & $17 / 20$ \\
To plan housing, schooling etc. & $17 / 20$ \\
For physiotherapy & $17 / 20$ \\
To make good memories & Yes \\
3. Avoid diagnostic delay & Yes \\
4. Avoid wrongful attributions to early symptoms & Yes \\
5. More positive profile (anxiety, wellbeing, & \\
adjectives) when boys are socially aware & \\
\hline
\end{tabular}

\begin{tabular}{lll}
$\begin{array}{l}\text { Table } 6 \\
\text { screening }\end{array}$ & Potential disadvantages of newborn \\
\hline & Screened & Transient \\
\hline 1. Decision regret after test & $1 / 20$ & $1 / 18$ \\
2. Lingering doubts about test result & N/A & $2 / 18$ \\
3. Mother-baby relationship & No & No \\
FIRST scores & No & No \\
Reject statement & No & No \\
Protect statements (protect/watch/trust/hugs) & Change & Change \\
Use of baby adjectives & noted & noted \\
& Slight & No \\
4. Increased anxiety (State) & No & No \\
5. Reduced wellbeing (GHQ 12) & & \\
\hline
\end{tabular}

GHQ, General Health Questionnaire.

seven healthy girls, the termination of four fetuses carrying the DMD-causing mutation, and one termination because of a chromosomal abnormality.

Sixteen of the 20 index families $(80 \%)$ reported that their reproductive patterning had changed as a result of the diagnosis. Fifteen of these 16 families had wanted more children but, after diagnosis, four decided against another pregnancy and 11 delayed. This delay is reflected in the birth interval: the mean interval for the screened cohort between first and second pregnancy was 41 months compared with 29 months for mothers in the general population. ${ }^{21}$ We cannot quantify how many affected boys might have been born to these 15 mothers, 10 of whom were high risk or obligate carriers, which illustrates the difficulty of using altered reproductive planning as an outcome measure.

Before diagnosis, 10 siblings were born to the later clinically diagnosed cohort (five unaffected boys, five girls), the unaffected boys all being born to women at low risk. The later clinically diagnosed cohort would have included two families with second affected children, but these were excluded because a younger brother had been diagnosed ( first), through newborn screening.

\section{DISCUSSION}

Eventual diagnosis is inevitable for families who have a boy with DMD. The purpose of our study was to identify the advantages and disadvantages of it being made in the newborn period (tables 5 and 6).

We have shown that most families modified their reproductive plans and had prenatal testing. This finding is very different from that reported by Hildes et $a l^{22}$ and could reflect the integrated service that is provided in Wales. In addition, most of those in the screened and later clinically diagnosed cohorts were in favour of earlier diagnosis on the grounds of gaining time to prepare emotionally and practically. Mothers from the later clinically diagnosed cohort perceived the advantage of avoiding diagnostic delay and expressed their regret about misunderstanding their son's early symptoms.

The profile of the screened cohort (first interview) and the later clinically diagnosed cohort is similar in terms of increased anxiety levels, reduced wellbeing scores, and more negative choice of descriptors. By the second interview, when boys from the screened cohort were more socially aware of their problem, maternal anxiety and wellbeing had returned to normal and descriptors were more positive than for the later clinically diagnosed cohort. Some mothers from both groups saw this difference as an advantage as they felt it was better to have faced the diagnosis when he was younger than learn about it when he was symptomatic and asking questions.

The case for newborn screening must take into account whether there are evident disadvantages that might outweigh these advantages. One potential disadvantage is decision regret after the test, which occurred in two cases (one each in the screened and transient cohorts), where informed consent to screening had not been optimal. We have addressed this issue recently. ${ }^{23}$ The study also highlighted the potential disadvantage of lingering doubts about the test result by those in the transient cohort. Our information leaflet is now more explicit about the occurrence of transient results, and the protocol recommends that each family is given a written record of venous blood creatine kinase levels, compared with potential levels in DMD, and health visitors are made more aware that lingering doubts may occur.

Concerns about the negative effect of newborn screening on the early mother-baby relationship have not been substantiated. We found no evidence, from either health visitor assessments (FIRST scores) or mothers' responses to the rejection/ protection index of rejection. There was also no evidence of any difference during the first year of life on any of the overprotection scores.

The analysis of baby descriptors has shown that there was no between cohort difference in usage of the negative descriptors by mothers of boys aged 6-9 months. The mothers in the screened and transient cohorts, however, were less likely to use certain positive descriptors and more likely to choose "cuddly". It is debatable whether this can be classified as a disadvantage because there was no evidence of rejection or overprotection on other measures. The qualitative accounts indicate that after diagnosis, mothers in the screened group felt that their sons had become "more precious". Therefore, we argue that this measure is very sensitive and has highlighted an important qualitative change in the mother-baby relationship.

We found evidence of slightly increased anxiety in both the screened and later clinically diagnosed cohorts in the year after diagnosis, but scores for the screened cohort were within the normal range by second interview. There was no evidence from anxiety scores that the transient cohort had suffered any disadvantage, and their wellbeing was in line with the control group, as was that of the screened cohort.

\section{Conclusion}

We have provided unique prospective data from families and health professionals on the psychosocial implications of newborn screening for DMD. On the basis of this evidence, we argue that a case can be made for newborn screening, subject to four conditions:

(1) The screening test must only be offered on the basis of informed parental choice.

(2) The programme should be accompanied by a rigorous protocol that details how the initial query is to be raised and ensures that there is continuing support for all identified families. 
(3) An infrastructure should be established that ensures communication and cooperation between primary, secondary, and tertiary support services, in particular an integrated genetic counselling service, a paediatric support service, and a designated person in newborn screening responsible for coordination.

(4) There is regular feedback from families and primary healthcare teams to ensure that the programme is being delivered in an acceptable way.

In view of recent concerns about the persisting late diagnosis of DMD, we believe it is now the time for other centres to review our evidence and consider introducing newborn screening for DMD.

\section{ACKNOWLEDGEMENTS}

We would like to thank all the families, midwives, health visitors, paediatricians, family care officers, physiotherapists, genetic nurse specialists, and general practitioners who participated in the research. We would also like to thank Claire Batchelor and Carolyn Eason for data collection, Dr Jane Fenton-May for information on reproductive patterning, the genetics team from the South West of England, including Dr Peter Lunt, Judith Howells, and Jan Smith, for information on some families with a later clinical diagnosis, and Charles Laugharne and Professor Patricia Lyne, for their constructive input to previous drafts of this paper. Finally we would like to thank the staff from the newborn screening laboratory for their technical support. This research received financial support from the Muscular Dystrophy Campaign of Great Britain, the Wellcome Trust, and the Wales Office of Research and Development.

\section{Authors' affiliations}

E P Parsons, School of Nursing and Midwifery Studies, University of Wales College of Medicine, Cardiff, UK

A J Clarke, Institute of Medical Genetics, University of Wales College of Medicine

K Hood, Division of General Practice, University of Wales College of Medicine

E Lycett, Family and Child Psychology Research Centre, City University, London, UK

D M Bradley, Department of Medical Biochemistry, University Hospital of Wales, Cardiff, UK

\section{REFERENCES}

1 Appleton RE, Nicolaides P. Early diagnosis of Duchenne muscular dystrophy. Lancet 1994;345:1243-4.
2 Bushby KMD, Hill A, Steele JG. Failure of early diagnosis in symptomatic Duchenne muscular dystrophy. Lancet 1999;353:557-8.

3 Zalaudek I, Bonelli RM, Koltringer $P$, et al. Early diagnosis in Duchenne muscular dystrophy. Lancet 1999;353:1975.

4 Firth M, Gardner-Medwin D, Hosking G, et al. Interviews with parents of boys suffering from Duchenne muscular dystrophy. Dev Med Child Neurol 1983;25:466-71.

5 Marshall PD, Galasko CSB. No improvement in delay of diagnosis of Duchenne muscular dystrophy. Lancet 1995;345:590-1.

6 Gardner-Medwin D, Bundey S, Green S. Early diagnosis of Duchenne muscular dystrophy. Lancet 1978;1:1 102.

7 Smith RA, Rogers $M$, Bradley DM, et al. Screening for Duchenne muscular dystrophy. Arch Dis Child 1989:64:1017-21.

8 Zellweger H, Antonik A. Newborn screening for Duchenne muscular dystrophy. Pediatrics 1975;55:30-4.

9 Bradley DM, Parsons EP. Newborn screening for Duchenne muscular dystrophy. Semin Neonatol 1998;3:27-34.

10 Al-Jader LN, Goodchild MC, Ryley HC, et al. Attitudes of parents of cystic fibrosis children towards neonatal screening and antenatal diagnosis. Clin Genet 1990;38:460-5.

11 Andrews LB, Fullarton JE, Holtzman NA, et al. Assessing genetic risks: implications for health and social policy. Washington DC: National Academy Press, 1994.

12 Smith RA, Rogers M, Bradley DM, et al. Screening for Duchenne muscular dystrophy. Arch Dis Child 1989;64:1017-21.

13 Bradley DM, Parsons EP, Clarke AJ. Experience with screening newborns for Duchenne muscular dystrophy in Wales. BM 1993;306:357-60.

14 Parsons EP, Bradley DM, Clarke AJ. Disclosure of Duchenne muscular dystrophy after newborn screening. Arch Dis Child 1996;74:550-3.

15 Parsons EP, Bradley DM. Complementary methodologies in the evaluation of newborn screening for Duchenne muscular dystrophy. In: Clarke AV, ed. The genetic testing of children. Oxford: Bioscientific, 1998:211-21.

16 Salariya EM, Cater JI. Mother-child relationship: FIRST score. J Adv Nurs 1984;9:589-95.

17 Green JM, Richards MPM, Kitzinger JV, et al. Mothers' perceptions of their 6-week-old babies: relationships with antenatal, intrapartum and postnatal factors. Irish Journal of Psychology 1991;12:133-44.

18 Speilberger CD. Manual for the State-Trait Personality Inventory STAI (Form Y). Palo Alto, CA: Consulting Psychologists Press, 1983

19 Marteau TM, Bekker $\mathrm{H}$. The development of a six-item short-form of the state scale of the Spielberger state-trait anxiety inventory (STAI). Br J Clin Psychol 1992;31:301-6.

20 Goldberg DP. The detection of psychiatric illness by questionnaire. London: Oxford University Press, 1972

21 Office for National Statistics. Birth statistics series FM1, no. 26, summary. London: HMSO, 1997.

22 Hildes E, Jacobs HK, Cameron A, et al. Impact of genetic counselling after neonatal screening for Duchenne muscular dystrophy. J Med Genet 1993;30:670-4.

23 Parsons EP, Clarke AJ, Hood K, et al. Feasibility of a change in service delivery: the case of optional newborn screening for Duchenne muscular dystrophy. Community Genetics 2000;3:17-23. 